\title{
Epidemiologia e etiologia das dermatofitoses em Goiânia, GO, Brasil
}

\author{
Epidemiology and etiology of dermatophytosis in Goiânia, GO, Brazil
}

\begin{abstract}
Milce Costa ${ }^{1}$, Xisto Sena Passos ${ }^{1}$, Lúcia Kioko Hasimoto e Souza ${ }^{1}$, André Thiago Borges Miranda ${ }^{1}$, Janine de Aquino Lemos ${ }^{1}$, Juldásio Galdino de Oliveira Júnior ${ }^{1}$ e Maria do Rosário Rodrigues Silva ${ }^{1}$
\end{abstract}

\begin{abstract}
Resumo Os dermatófitos são um grupo de fungos taxonomicamente relacionados que têm a capacidade de invadir os tecidos queratinizados (pele, pêlo e unha) dos homens e animais produzindo infecções denominadas dermatofitoses. Com o intuito de avaliar a epidemiologia e etiologia das infecções causadas por estes fungos em Goiânia, GO, foram examinadas no Laboratório de Micologia do Instituto de Patologia Tropical e Saúde Pública da Universidade Federal de Goiás, de janeiro a dezembro de 1999, 1.955 amostras de indivíduos com suspeita clínica de dermatofitoses. Foram isolados $445(22,8 \%)$ cepas de dermatófitos e identificados principalmente Trichophyton rubrum (49,4\%), Trichophyton mentagrophytes (30,8\%) e Microsporum canis (12,6\%). Quanto à localização das lesões, os membros inferiores, unhas dos pés e couro cabeludo foram as regiões mais acometidas. Neste estudo foram avaliados dados correlacionados a sexo, faixa etária, local das lesões e agente etiológico.
\end{abstract}

Palavras-chaves: Dermatofitoses. Etiologia. Epidemiologia.

Abstract Dermatophytes are a group of closely related fungi that have the capacity to invade keratinized tissue (skin, hair, and nails) of humans and animals to produce infections called dermatophytosis. In order to review the etiology and epidemiology of dermatophytosis in Goiânia, GO, 1955 specimens with diagnostic suspicion of dermatophytic lesions, were collected from January to December, 1999, from the Mycology Laboratory in the Institute of Tropical Pathology and Public Health, Federal University of Goiás. A total of 445 (22.8\%) samples were positive for dermatophytes and Trichophyton rubrum was the most frequently isolated species (49.4\%) followed by Trichophyton mentagrophytes (30.8\%) and Microsporum canis (12.6\%). Concerning the location of the lesions, the inferior limbs, feet and nails together with tinea capitis were the most frequently found clinical pattern in the majority of patients. Correlation between sex, age, location of the lesions and etiologic agents is considered in the study.

Key-words: Dermatophytosis. Etiology. Epidemiology.

As dermatofitoses são infecções superficiais cutâneas produzidas por fungos queratinofílicos denominados dermatófitos, que compreendem os gêneros Trichophyton, Microsporum e Epidermophyton. Estes agentes invadem o extrato córneo da pele e de outros tecidos queratinizados dos homens e animais produzindo as chamadas tinhas. Estima-se que 10 a $15 \%$ da população humana poderá ser infectada por estes microrganismos no decorrer de sua vida ${ }^{14} 1720$.
Os dermatófitos apresentam uma predileção ecológica, no que diz respeito à sua adaptação ao meio ambiente, podendo ser divididos em 3 grandes grupos, em relação ao seu habitat, sendo classificados como geofílicos, zoofílicos e antropofílicos ${ }^{20}$.

Quanto à distribuição geográfica, os dermatófitos são ubiquitários, não havendo área ou grupo de pessoas que se encontrem isoladas destes fungos ${ }^{16}$. A variada distribuição etiológica das dermatofitoses

1. Instituto de Patologia Tropical e Saúde Pública (IPTSP) da Universidade Federal de Goiás (UFG), Goiânia, GO.

Endereço para correspondência: Profa. Maria do Rosário Rodrigues Silva. IPTSP/ UFG. Av. Delenda Resende de Melo esq.com 1ª. Av. Setor Universitário,

Caixa Postal 131, 74605-050 Goiânia, GO

Tel: 5562 261-6497; Fax: 5562 202-3066

e-mail: rosario@iptsp.ufg.br

Recebido para publicação em 17/5/2001 
pode ser explicada por áreas onde condições geoclimáticas e sociais são extremamente diferenciadas, fatalmente influenciando nas espécies de dermatófitos isolados ${ }^{20}$.

A freqüência e etiologia das dermatofitoses têm sido descritas em Goiânia, GO, por diversos autores, observando-se diferenças na etiologia dos agentes ao longo dos anos ${ }^{3411}$. O presente trabalho tem como objetivo contribuir para o conhecimento da freqüência e etiologia das dermatofitoses ocorridas em Goiânia, GO, no período de janeiro a dezembro de 1999.

\section{MATERIAL E MÉTODOS}

De janeiro a dezembro de 1999 , foram coletadas no Laboratório de Micologia do Instituto de Patologia Tropical e Saúde Pública da Universidade Federal de Goiás, 1.955 amostras de diferentes regiões do corpo com suspeita clínica de infecção fúngica, sendo registrados dados pessoais dos pacientes como idade, sexo e localização das lesões.

As amostras para pesquisa de dermatófitos foram obtidas de pele, pêlo e unha. Do couro cabeludo, o pêlo parasitado foi retirado com pinça de depilação estéril e das outras regiões foram coletadas mediante raspagem com bisturi. Para padronização na compilação de dados para os dermatófitos, as diferentes regiões do corpo foram agrupadas de tal modo que foram consideradas as seguintes regiões: a) couro cabeludo; b) face e tronco (face, orelha, pescoço, axila, tórax, costas e abdome); c) membros superiores (antebraço, cotovelo, braço e regiões dorsal, palmar e interdigital das mãos); d) inguinocrural (virilha e nádegas); e) membros inferiores (coxa, joelho, perna e regiões dorsal, plantar e interdigital dos pés); f) unhas das mãos; g) unhas dos pés.

O exame micológico direto de todos os materiais coletados foi realizado com $\mathrm{KOH}$ a $40 \%$ e o isolamento das amostras foi feito em ágar Sabouraud dextrose, ágar batata e ágar seletivo para fungos patogênicos mantidos à temperatura ambiente por no mínimo 30 dias.

A identificação dos gêneros e espécies de dermatófitos foi baseada nas características macro e microscópicas de suas colônias bem como através de microcultivo em lâmina. Provas adicionais como o teste de perfuração do pêlo in vitro e prova da utilização da uréia foram utilizadas para diferenciar T. mentagrophytes de T. rubrum.

\section{RESULTADOS}

Dos 1.955 espécimes coletados de pacientes com suspeita clínica de infecção fúngica, de janeiro a dezembro de 1999, foram isolados e identificados 445 (22,8\%) amostras positivas para dermatófitos. Das amostras positivas, $241(54,2 \%)$ foram provenientes de indivíduos do sexo masculino e 204 (45,8\%) do sexo feminino.

Lesões por dermatófitos foram mais freqüentemente observadas nos membros inferiores (pés e pernas), com
$143(32,1 \%)$ em 445 casos, sendo mais freqüentes em indivíduos entre 21 e 30 anos. Lesões das unhas dos pés também foram freqüentemente verificadas, sendo encontrados $78(17,5 \%)$ dos 445 casos, com maior número registrado nos indivíduos de 41 a 50 anos, enquanto $14,1 \%$ das lesões do couro cabeludo foram observados principalmente em crianças menores de 10 anos. A localização das lesões correlacionada com a idade dos pacientes encontra-se na Tabela 1.

Tabela 1 - Correlação da localização das lesões com a faixa etária dos pacientes.

\begin{tabular}{|c|c|c|c|c|c|c|c|c|c|c|c|c|c|c|c|}
\hline \multirow[b]{3}{*}{ Local da lesão } & \multicolumn{14}{|c|}{ Faixa etária } & \multirow{3}{*}{$\begin{array}{c}\text { Total } \\
\mathrm{n}\end{array}$} \\
\hline & \multicolumn{2}{|c|}{$0-10$} & \multicolumn{2}{|c|}{$11-20$} & \multicolumn{2}{|c|}{$21-30$} & \multicolumn{2}{|c|}{$31-40$} & \multicolumn{2}{|c|}{$41-50$} & \multicolumn{2}{|c|}{$51-60$} & \multicolumn{2}{|c|}{$>60$} & \\
\hline & $\mathrm{n}$ & $\%$ & $\mathrm{n}$ & $\%$ & $\mathrm{n}$ & $\%$ & $\mathrm{n}$ & $\%$ & $\mathrm{n}$ & $\%$ & $\mathrm{n}$ & $\%$ & $\mathrm{n}$ & $\%$ & \\
\hline $\mathrm{CC}$ & 60 & 95,2 & 3 & 4,8 & - & - & - & - & - & - & - & - & - & - & 63 \\
\hline Face, tronco & 10 & 23,2 & 7 & 16,3 & 11 & 25,6 & 8 & 18,6 & 5 & 11,6 & 2 & 4,6 & - & - & 43 \\
\hline MMSS & 7 & 22,6 & 1 & 3,2 & 13 & 41,9 & 4 & 12,9 & 2 & 6,4 & 3 & 9,7 & 1 & 3,2 & 31 \\
\hline IC & - & - & 13 & 20,0 & 24 & 36,9 & 14 & 21,5 & 10 & 15,4 & 3 & 4,6 & 1 & 1,5 & 65 \\
\hline MMII & 3 & 2,1 & 16 & 11,2 & 37 & 25,8 & 23 & 16,1 & 34 & 23,8 & 11 & 7,7 & 19 & 13,3 & 143 \\
\hline Unhas mãos & - & - & 5 & 22,7 & 5 & 22,7 & - & - & 1 & 4,5 & 4 & 18,2 & 7 & 31,8 & 22 \\
\hline Unhas pés & - & - & - & - & 7 & 9 & 8 & 10,2 & 28 & 35,9 & 18 & 23,1 & 17 & 21,8 & 78 \\
\hline Total & 80 & 18,0 & 45 & 10,1 & 97 & 21,8 & 57 & 12,8 & 80 & 18,0 & 41 & 9,2 & 45 & 10,1 & 445 \\
\hline
\end{tabular}

$\mathrm{CC}=$ couro cabeludo; $\mathrm{MMSS}=$ membros superiores; $\mathrm{IC}=$ inguino crural; $\mathrm{MMII}=$ membros inferiores

Em ordem de freqüência foram isolados: T. rubrum observado em 220 (49,4\%) lesões; T. mentagrophytes em $137(30,8 \%)$; M. canis em $56(12,6 \%)$; E. floccosum em $17(3,8 \%)$ e $T$. tonsurans em $5(1,1 \%)$, não sendo possível a identificação da espécie em $10(2,2 \%)$ casos.

No que se refere à etiologia das lesões, nas diferentes regiões do corpo, foi verificado em 143 casos que T. rubrum e T. mentagrophytes foram os agentes que mais acometeram a região dos membros inferiores com $77(53,8 \%)$ e 48 (33,6\%) casos, respectivamente. T. rubrum foi responsável pelo maior número de lesões nas unhas dos pés e na região inguino-crural perfazendo um total de 60,2\% (47/78) e de $55,4 \%$ (36/65), respectivamente. M. canis foi o agente que mais acometeu o couro cabeludo, com $60,3 \%(38 / 63)$ dos casos. 
Com relação ao sexo dos pacientes e às localizações das lesões, verificou-se que havia um predomínio de lesões no couro cabeludo e região inguino-crural no sexo masculino, no entanto nas unhas dos pés ocorreu um maior número de casos no sexo feminino. A Tabela 2 mostra a etiologia das dermatofitoses correlacionados ao sexo e localização das lesões.
Fazendo-se a correlação do agente etiológico com a faixa etária, verificou-se que $M$. canis foi o responsável pela maioria das lesões em menores de 10 anos, sendo que $T$. rubrum e T. mentagrophytes prevaleceram na fase adulta. Os dados da etiologia e idade dos pacientes encontram-se na Tabela 3.

Tabela 2 - Correlação da etiologia das dermatofitoses com o local da lesão e sexo dos pacientes.

\begin{tabular}{|c|c|c|c|c|c|c|c|c|c|c|c|c|c|c|}
\hline \multirow[b]{3}{*}{ Etiologia } & & & \multirow{2}{*}{\multicolumn{2}{|c|}{ Face tronco }} & \multirow{2}{*}{\multicolumn{2}{|c|}{ MMSS }} & \multirow{2}{*}{\multicolumn{2}{|c|}{ IC }} & & & \multicolumn{4}{|c|}{ Unhas } \\
\hline & \multicolumn{2}{|c|}{$\mathrm{CC}$} & & & & & & & \multicolumn{2}{|c|}{ MMII } & \multicolumn{2}{|c|}{ mãos } & \multicolumn{2}{|c|}{ pés } \\
\hline & $M$ & $\mathrm{~F}$ & $M$ & $\mathrm{~F}$ & $M$ & $F$ & $M$ & $\mathrm{~F}$ & $M$ & $\mathrm{~F}$ & $M$ & $\mathrm{~F}$ & $M$ & $\mathrm{~F}$ \\
\hline T. rubrum & 9 & - & 10 & 10 & 12 & 6 & 30 & 6 & 37 & 40 & 13 & - & 10 & 37 \\
\hline T. mentagrophytes & 3 & 6 & 10 & 4 & 5 & 5 & 14 & 6 & 29 & 19 & 4 & 5 & 10 & 17 \\
\hline M. canis & 23 & 15 & 3 & 4 & 1 & 2 & 2 & - & 1 & 5 & - & - & - & - \\
\hline E. floccosum & - & - & - & 2 & - & - & 3 & 4 & 4 & 2 & - & - & - & 2 \\
\hline T.tonsurans & 3 & 2 & - & - & - & - & - & - & - & - & - & - & - & - \\
\hline Trichophyton sp & - & - & - & - & - & - & - & - & 1 & 3 & - & - & - & 2 \\
\hline Microsporum sp & 2 & - & - & - & - & - & - & - & 2 & - & - & - & - & - \\
\hline Total & 40 & 23 & 23 & 20 & 18 & 13 & 49 & 16 & 74 & 69 & 17 & 5 & 20 & 58 \\
\hline
\end{tabular}

$\mathrm{CC}=$ couro cabeludo; $\mathrm{MMSS}=$ membros superiores; $\mathrm{IC}=$ inguino-crural; $\mathrm{MMII}=$ membros inferiores.

Tabela 3 - Etiologia das dermatofitoses segundo a idade dos pacientes.

\begin{tabular}{|c|c|c|c|c|c|c|c|c|c|c|c|c|c|c|c|}
\hline \multirow[b]{3}{*}{ Etiologia } & \multicolumn{14}{|c|}{ Faixa etária } & \multirow{3}{*}{ total } \\
\hline & \multicolumn{2}{|c|}{$0-10$} & \multicolumn{2}{|c|}{$11-20$} & \multicolumn{2}{|c|}{$21-30$} & \multicolumn{2}{|c|}{$31-40$} & \multicolumn{2}{|c|}{$41-50$} & \multicolumn{2}{|c|}{$51-60$} & \multicolumn{2}{|c|}{$>60$} & \\
\hline & $\mathrm{n}$ & $\%$ & $\mathrm{n}$ & $\%$ & $\mathrm{n}$ & $\%$ & $\mathrm{n}$ & $\%$ & $\mathrm{n}$ & $\%$ & $\mathrm{n}$ & $\%$ & $\mathrm{n}$ & $\%$ & \\
\hline T. rubrum & 9 & 4,1 & 19 & 8,6 & 53 & 24,1 & 38 & 17,3 & 43 & 19,5 & 27 & 12,3 & 31 & 14,1 & 220 \\
\hline T. mentagrophytes & 17 & 12,4 & 16 & 11,7 & 35 & 25,5 & 13 & 9,5 & 30 & 21,9 & 12 & 8,7 & 14 & 10,2 & 137 \\
\hline M. canis & 47 & 83,9 & 5 & 8,9 & 2 & 3,6 & 2 & 3,6 & - & - & - & - & - & - & 56 \\
\hline E. floccosum & - & - & 5 & 29,4 & 3 & 17,6 & 2 & 11,8 & 5 & 29,4 & 2 & 11,8 & - & - & 17 \\
\hline T.tonsurans & 5 & 100 & - & - & - & - & - & - & - & - & - & - & - & - & 5 \\
\hline Trichophyton sp & - & - & - & - & 4 & 66,7 & 2 & 33,3 & - & - & - & - & - & - & 6 \\
\hline Microsporum $s p$ & 2 & 50 & - & - & - & - & - & - & 2 & 50 & - & - & - & - & 4 \\
\hline Total & \multicolumn{2}{|c|}{80} & \multicolumn{2}{|c|}{45} & \multicolumn{2}{|c|}{97} & \multicolumn{2}{|c|}{57} & \multicolumn{2}{|c|}{80} & \multicolumn{2}{|c|}{41} & \multicolumn{2}{|c|}{45} & 445 \\
\hline
\end{tabular}

\section{DISCUSSÃO}

Dos 1.955 pacientes examinados no laboratório de Micologia da Universidade Federal de Goiás em Goiânia, GO durante o período de um ano, foram diagnosticados $445(22,8 \%)$ casos de dermatofitoses. Este elevado índice de infecção por dermatófitos também tem sido descrito por diferentes pesquisadores. No Espírito Santo, Mattêde et al $^{13}$ verificaram dermatofitoses em $50,2 \%$ de 470 pacientes atendidos no Hospital Universitário de Vitória, ES. Na região Sul, Lopes et al ${ }^{10}$ detectaram $1.113(20,7 \%)$ casos de dermatofitoses isolados de um total de 5.373 pacientes atendidos no Serviço de Micologia do Hospital Universitário de Santa Maria, RS.

A etiologia destas micoses apresenta uma grande variabilidade nas diferentes regiões do $\mathrm{Brasil}^{2}{ }^{12}{ }^{13}$. As constantes correntes migratórias que ocorrem no país têm sido citadas como uma das principais causas para a alteração desta etiologia ${ }^{5789}$.

$\mathrm{O}$ agente predominante em nossos resultados causando dermatofitoses em $220(49,4 \%)$ dos 445 casos foi $T$. rubrum, o qual também tem sido isolado como a principal causa de dermatofitoses, observado por numerosos pesquisadores no Brasil e no mundo 1014151618 . Em nossa região, Machado et $\mathrm{al}^{11} \mathrm{e}$ Costa et al ${ }^{3}$ relataram T. rubrum como a espécie predominante nos períodos de 1967 a 1973 e de 1993 a 1997, respectivamente, enquanto Fernandes et al ${ }^{4}$ verificaram a predominância de T. mentagrophytes em estudo realizado entre 1984 a 1990.

A elevada porcentagem de lesões por dermatófitos nos pés e unhas dos pés causadas predominantemente por $T$. rubrum entre indivíduos de 20 a 50 anos, encontrada no nosso estudo é justificada por Mazón et a ${ }^{14}$ que consideram que as lesões ocorram nesta localização e nesta faixa etária principalmente devido ao aumento de práticas desportivas nesta idade, concomitante ao uso de sapatos fechados. Com relação à etiologia predominante nos pés, $T$. rubrum também predomina na maioria das casuísticas estudadas por outros autores ${ }^{1141619}$. 
O achado de lesões no couro cabeludo em menores de 10 anos (60/63), tendo como agente etiológico $M$. canis na maioria dos casos encontrados, pode ocorrer devido ao maior contato das crianças com animais, principalmente cães e gatos, habitat natural desta espécie. Este agente etiológico tem sido a principal causa de lesões do couro cabeludo em crianças $^{34}{ }^{16}$. De acordo com dados obtidos em 1992 na mesma região geográfica do presente estudo, foi verificado uma elevada percentagem de $T$. tonsurans $(15,4 \%)$ no couro cabeludo ${ }^{4}$, no entanto, a porcentagem obtida no presente trabalho foi de apenas $7,9 \%$ mostrando que houve diversificação na etiologia destas lesões nesta localização do corpo.

Diante do exposto, podemos concluir que o conhecimento da etiologia, o qual se relaciona com o habitat natural dos agentes (geofílicos, antropofílicos e zoofílicos) provavelmente seja a principal meta para evitar a propagação das dermatofitoses. Sugerimos portanto, que estudos prospectivos da etiologia destas doenças devam ser realizados continuamente, de modo a contribuir no controle da infecção.

\section{REFERÊNCIAS BIBLIOGRÁFICAS}

1. Boncompte E, Algueró M, Videla S, Forn J. Contribuición al estudio de las dermatomicosis en Cataluña. Revista Iberoamericana de Micologia 14:26-28, 1997.

2. Carvalho MTF, Furtado T, Pereira CAC. Dermatófitos geofílicos em Juiz de Fora. Revista de Dermatologia 10:99-106, 1983.

3. Costa TR, Costa MR, Silva MRR, Silva MV, Rodrigues AB, Fernandes ODL, Soares AJ, Silva MJ. Etiologia e epidemiologia das dermatofitoses em Goiânia-GO. Revista da Sociedade Brasileira de Medicina Tropical 32:367-371, 1999.

4. Fernandes OFL, Silva MRR, Vilela NA, Silva ER, Silva HM, Jesuíno RS. Etiologia das dermatofitoses em pacientes atendidos no laboratório de Micologia do Instituto de Patologia Tropical e Saúde Pública da Universidade Federal de Goiás. Revista de Patologia Tropical 21:189-197, 1992.

5. Fernandez TE, Fernandez CC. Variacion de la frecuencia de los agentes etiologicos de la Tinea capitis en Guayaquil-Ecuador. Revista Ecuatoriana de Higiene y Medicina Tropical 34:51-53, 1984.

6. Florencio VD, Balmas JAR. Cambios en la epidemiología de las tiñas. Aspectos particulares de Andalucía. Revista Iberoamericana de Micologia 16:3-6, 1999.

7. Furtado MSS, Ihara LT, Maroja MF. Tinea capitis na cidade de Manaus-AM. Anais Brasileiro de Dermatologia 60:315-318, 1985.

8. Gambale W, Paula CR, Correa B, Purchio A. Incidência de micoses superficiais em São Paulo-SP. Anais Brasileiro de Dermatologia 62: 193-194, 1987.

9. Gianelli M, Araujo MAR, Proença NG, Zaitz C. Dermatofitose do pé: estudo epidemiológico prospectivo. Anais Brasileiro de Dermatologia 63:9-12, 1988.

10. Lopes JO, Alves SH, Benevenga JP. Dermatofitoses humanas no interior do Rio Grande do Sul no período 1998-1992. Revista do Instituto de Medicina Tropical São Paulo 14:115-119, 1994.
11. Machado OP, Rodrigues MR, Souza MHR. Ocorrência de dermatófitos em Goiás. Revista de Patologia Tropical 3:273-276, 1974.

12. Martini JPJ, Souza LCD, Costa HC. Dermatófitos isolados em pacientes do Hospital "Lauro de Souza Lima". Bauru, SP. Salustra 6:1-6, 1987.

13. Mattêde MGS, Coelho CC, Mattede AF, Perin FC, Junior LP. Etiologia das Dermatofitoses em Vitória- ES. Anais Brasileiro de Dermatologia 61:177-182, 1986.

14. Mazón A, Salvo S, Vives R, Valcayo A, Sabalza MA. Estudio etiologico y epidemiologico de las dermatofitosis en Navarra (España). Revista Iberoamericana de Micologia 14:65-68, 1997.

15. Mezzari A. Frequency of dermatophytes in the metropolitan area of Porto Alegre, RS, Brazil. Revista do Instituto de Medicina Tropical de São Paulo 40:71-76, 1998.

16. Palacio A, Cuétara MS, Valle A, Gonzáles A, Almondaraim I, Castillo MJR, Vasallo AM, Miguens MP. Cambios epidemiológicos observados en un decenio en las dermatofitosis del hospital universitario "12 de Octubre" de Madrid: nuevas especies emergentes. Revista Iberoamericana Micologia 16:101-106, 1999.

17. Rubio MC, Rezusta A, Tomás JG, Ruesca RB. Perspectiva micológica de los dermatofitos en el ser humano. Revista lberoamericana de Micologia 16:16-22, 1999.

18. Santos JI, Negri CM, Wagner DC. Some aspects of dermatophytes seen at University Hospital in Florianópolis, Santa Catarina, Brazil. Revista do Instituto Medicina Tropical de São Paulo 39:137-140, 1997.

19. Sberna F, Farella V, Geti V, Taviti F, Agostini G, Vanini P, Knöpfel B, Difonzo EM. Epidemiology of the dermatophytoses in the Florence area of Italy: 1985-1990. Mycopathologia 12:153-162, 1993.

20. Sidrim JJC, Diógenes MJN, Paixão GC. Dermatofitose. Fundamentos Clínicos e Laboratoriais da Micologia Médica 12:107-131, 1999. 